\title{
Discussion on the Application of Sensor Technology in Mechanical and Electronic Industry
}

\author{
Jinhong Zou ${ }^{1, a}$, Zhenghao Jian ${ }^{2}$ \\ ${ }^{1,2}$ School of mechanical and Vehicular Engineering, Nanchang Institute of Technology \\ aZouJinhong@163.com
}

Keywords: sensor, Mechanical and electronic technology, Mechatronics

\begin{abstract}
In today's era of rapid development of science and technology, the various disciplines have made considerable progress, at the same time, the subject and the crossesbetween subject and discipline are more and more ,and this cross becomes more and more complex and diverse. Among them, the mechanical and electronic engineering is a typical interdisciplinary subject.The Mechanical and electronic engineering discipline is based on mechanical manufacturing,joined the product of the integration of electronic technology.The sensor technology is an important part in mechanical and electronic engineering industry,and it can greatly improve the overall operation efficiency and has the advantage of low cost and high efficiency.At the same time, it can get the accurate and effective information in the development of the technology and enhance the ability of monitoring and management.This paper simply introduces the relevant technology of the sensor, and discusses the application of sensor in mechatronics. This paper tries to analyze the application status of sensor technology and the development direction of electronic technology in the mechanical and electronic industry.
\end{abstract}

\section{The correlation technique of sensor technology}

Nowadays, the information processing technology has achieved great development, microprocessor and computer technology has gained rapid development and promotion, in order to adapt to the development of these technologies, the development of sensor technology also has the corresponding development and promotion. The establishment and improvement of microprocessor and computer technology has enhanced the application scope and practical technology of these two technology in the modern production and life.The sensor is used as a front-end unit in information collection system, has gradually become the key device of mechanical and electronic automation and intelligence and its importance is more and more obvious in the development of mechanical and electronic systems.

In simple terms, a sensor is a device that transforms a physical quantity or chemical quantity into an electrical signal that is easy to record and transmit. It is a kind of pre unit device of mechanical electronic measurement system, which is used to convert the input variables which are difficult to measure and transport into the electrical signals which can be measured conveniently.It comprises a support body and a sensing element connected with the circuit and it is a integral part of the whole sensing system. The general sensor consists of a sensitive element, a switching element, a signal conditioning circuit and other auxiliary circuits, as shown in figure 1.

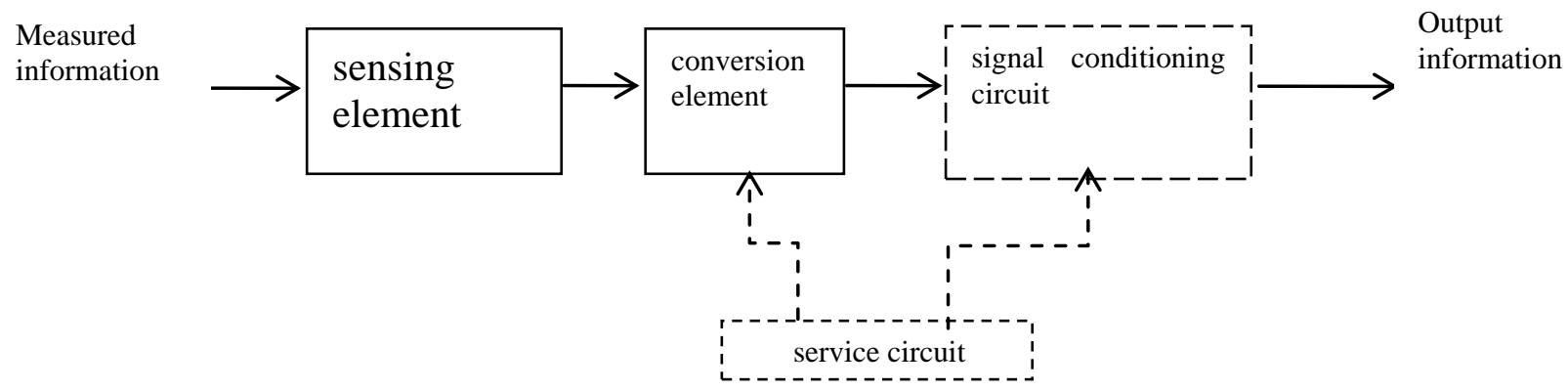

Figure 1 
Measured information first enter into the sensing element of sensor,the information signal amplitude is smaller and mixed with the noise and interference signal.After that, the signal will be shaped into a waveform with the best characteristics,this is in order to facilitate the signal processing. Sometimes, in order to transmit the signal, the signal will be linearized. This kind of work will be completed by the amplifier, filter and some analog circuits. Sensor is the most important component of the whole sensing system, which determines the performance of the sensing system to some extent.

The sensor's responsibility is translating a selected object or process to the amount of information available for measurement and transmission.The object of transformation can be solid, liquid and gas, the state of the object can be static or dynamic or static or association of activity and inertia,the initial state can be detected by different ways.According to the basic principle of the sensor, the characteristics of the input object and the corresponding information will be converted into electronic information and then the electrical signal is transmitted and measured for getting the information we want.

From different point of views, the sensor have different classification, as shown in Table 1.

Table 1 Sensor classification

\begin{tabular}{|l|l|}
\hline $\begin{array}{l}\text { Classification of measured } \\
\text { attributes:displacement, }\end{array}$ & $\begin{array}{l}\text { displacement, velocity, acceleration, pressure, } \\
\text { temperature, flow and other sensors. }\end{array}$ \\
\hline Classification of working principle & $\begin{array}{l}\text { Resistance-type,inductance-type,capacitance- } \\
\text { type, piezoelectric type, electromagnetic, } \\
\text { laser-type and photoelectric type or other } \\
\text { sensors. }\end{array}$ \\
\hline Classification of signal conversion feature & $\begin{array}{l}\text { Structural sensor (such as capacitance, } \\
\text { inductance sensor, etc.). } \\
\text { Physical sensors (such as magneto-electric, } \\
\text { photoelectric sensor etc.) }\end{array}$ \\
\hline Classification of output parameter & $\begin{array}{l}\text { Analog sensor, digital sensor } \\
\text { Analog sensor, digital sensor }\end{array}$ \\
$\begin{array}{l}\text { Parametric sensor (such as capacitance, } \\
\text { inductance sensor, etc.); } \\
\text { Power type sensors (such as piezoelectric, } \\
\text { magnetic sensor etc.) }\end{array}$ \\
\hline
\end{tabular}

With the rapid development of materials science, there are more and more sensors physical applications, among them, resistive transducer,capacitive transducer and magnetoelectric sensor are mostly be used in the measurement work. The working principle of the common physical sensor is not complicated, and the measurement conversion circuit is basically fixed and it is the most mature in application of the mechatronic engineering.

\section{Application of sensors in the development of mechanical and Electronic Engineering}

On the basis of the sensor's application characteristics and broad prospects for development,its application and importance in the field of mechanical and electronic engineering is gradually emerging .In the development of mechatronic engineering, electronic technology, automation control technology and machinery manufacturing technology have gradually been developed in different directions.In the process of the development of the core technology, the sensing technology plays an important role in the communication and integration. Therefore, the development prospects of the sensor in mechanical and electronic engineering also have different applications.

1) Intelligent direction

According to the mechanical field, especially in robot field,on the basis of characteristics of the object,the emergence of intelligence can make more specific image in line with the definition of intelligent behavior, which can adapt well to a variety of situations. In the early,the application of the sensor is to provide the basic information of the object, and then through the pattern recognition 
technology to achieve a primary intelligent application. In the later stage of development, the sensor can be used to process information for image, sound, temperature and other larger objects, and also can make the data transfer of information objects more accurate. Under the trend of intelligent,robot can collect and process information with large number, multi dimension and high precision data. The robot not only improve the ability of accurate identification,but also can carry out active learning, making the embodiment of intellectualization more obvious. In this process, Sensors play a large role in collecting and processing information.

2) Digital direction

The information has multi dimension and multi-level and diversified characteristics in reality, these complex information can achieve multi-directional collection and be transformed into a unified data format through the development and application of sensor technology,such as temperature, pressure, distance etc..These unified data will be further transformed into an accurate and measurable figure and be built a digital model.On the basis of the development of digital technology, the equipments of mechatronic engineering, such as robot and numerical control machine,can greatly improve the production efficiency and improve the production efficiency by using digital information and digital model.Therefore, the development of digital sensor technology will further enhance the development of mechatronic engineering technology digitization, high precision, high dimensions, and build a more practical and efficient digital model.

3) Network direction

Sensor devices are generally built into an integrated network port,and appeared in actual production application in the form of networking equipment.Sensor accesses to the Internet through 4G, 5G, WIFI, RFID, ZIGBEE, Bluetooth and other forms of transmission,providing information interface for remote monitoring, making monitor and operator can remotely monitor and control the mechanical and electronic equipment,which greatly break through the limitation of space and distance and let each sensor can be connected together and form the information network, so that a variety of mechanical and electronic devices can be interconnected with each other and work together to achieve data sharing between each other,improving the overall work efficiency. Sensor has achieved a breakthrough in the direction of the network,making mechanical and electronic equipment have become an important component of the Internet.

4) Modular direction

Along with the cross development and comprehensive development of mechanical and electronic engineering,mechanical and electronic equipment gradually evolved into the development direction of modularization.In the process of adapting to the modularization of mechanical and electronic equipment,the sensor, as an important part of mechanical and electronic equipment technology,Also gradually developed a suitable modular direction .Sensors on the one hand can be embedded in other module of mechanical and electrical equipment,providing distance measurement, temperature control and other functions to enhance the function of the module, on the other hand, different sensors can be combined together to integrate a multifunctional module,for example, the perception module of the robot, through the camera equipment, infrared devices, temperature sensing devices and other components of the robot perception module to provide a full range of information about the surrounding environment for robot.Thus, the modular development of the sensor in the development and application of mechanical and electronic equipment has great functions.

5) Humanized development

Along with the development needs of mechanical and electronic engineering technology in the modernization process,mechanical and electronic equipment is gradually tending to humanized design and manufacture. The sensor provides a powerful help for the development of mechanical and electronic equipment ,for example, if you take the gesture, voice and other forms of control and operation, you need the corresponding sensors to collect these complex information and transform it into a signal that can be measured and transmitted to the relevant detection and control equipment. The adoption of these new forms of interactive information transmission, mechanical and electronic equipment can break through a variety of restrictions to achieve a more diverse 
practical applications and meet the needs of people who live and work in different environments. In the design, manufacture and application of the robot, the humanized development of the sensor can also promote the robot to have a more comprehensive and accurate information acquisition to the external environment, so as to make a more humanized response.

6) Environmental friendly direction

In the practical application of mechanical and electronic equipment, sensors are also widely used to collect information about the operation of the mechanical and electrical equipment such as the wear of the equipment, the use of raw materials, equipment failure, pollutant emissions and so on.According to the information collected by the sensors, the equipment can be analyzed and tested, which can adjust the mechanical and electronic equipment properly and have an accurate understanding about the situation of the module control and further optimize the allocation of equipment resources.Taking the corresponding measures to the equipment in the view of the situation of serious wear and obvious aging phenomenon. According to the feedback data to develop appropriate maintenance or replacement plan to reduce emissions of pollutants to a minimum,improving the utilization rate of raw materials and to realize the environmental friendly development of mechanical and electronic equipment.

\section{The development and application of Mechatronics technology}

Modern mechatronics is not only refers to the combination of mechanical, electronic and information technology, but also includes the integration of optical and electrical, mechanical and electrical integration, mechanical and electrical integration, mechanical and electrical integration, etc.Mechatronics is the scientific thinking of the combination of various disciplines,highlighting the coordination and cooperation of various technologies in the manufacture of electromechanical products, and striving to achieve the optimization of the whole system.

Mechatronics is the product of the combination of a variety of technical disciplines, is not a simple technology superposition.In the practical design and manufacture, it is necessary to take into account the intrinsic connection and difference between the machine and the electronics, selecting the appropriate drive and production and testing equipment according to the actual production requirements, including the sensing equipment, information processing equipment, automatic control equipment, etc.. Compared with traditional electrical equipment,mechatronics equipment has more intelligent and efficient advantages, to the greater extent the product performance has been greatly improved and has met the actual needs of people.Mechanical and electrical integration products require sensors to obtain a large amount of information quickly and accurately and can minimize the impact of the external work environment, and to receive and process all kinds of parameters, working status, and other relevant information in the process of the project,to measure and analyze the converted information and then feedback to the control equipment,in order to realize the automatic control in the manufacture process of mechatronic products.

The sensor in the development of mechanical and electronic technology, also entered the development stage of integration and intelligence, not only the scope of receiving information is expanded,but also the device for detecting the information is having the performance of the signal to be filtered, amplified, converted and transmitted truly.The sensor technology puts the sensor and signal processing integrated circuit together,forming the sensor information,adding the microprocessor to the information sensor chip and the intelligent sensor is integrated.then it will have a broader space for development in the application of mechatronics.Sensor is a bridge to connect the real world with the mechanical and electronic equipment,whether it is on the detection of the surrounding environment and information collection or on the actual operation of the feedback, the information collected and converted by the sensor is very important to the operation of the equipment and provides an indispensable foundation for the development of mechanical and electronic technology.In summary, the sensor technology development has great development potential and application prospects in the development of machinery and electronics industry. 


\section{References}

[1] Qi changpu. Application of wireless sensor network based on ZigBee in monitoring system[D]. Wuhan University of Technology. 2008.

[2] Wang zhao. Research on Wireless Sensor Network Used in Condition Monitoring of Mechanical Equipment[D].Taiyuan University of Technology.2010.

[3] Rui yannian. Mechanical and electrical integration system design. Beijing Machinery Industry Press.2004.

[4] Zhang hao, Zhang xiliang, Zhou shichong, "Mechanical and electrical integration technology development and application" Beijing Agricultural Mechanization Research Press.2006.

[5] Zou huijun, Zhang qing, Tian zhibin. "Mechanical and electrical integration system conceptual design process model" Beijing Mechanical Design and Research Press.2002. 